\title{
Dietary patterns obtained through principal components analysis: the effect of input variable quantification
}

\author{
Andrew D. A. C. Smith ${ }^{1}$, Pauline M. Emmett ${ }^{1}$, P. K. Newby ${ }^{2,3,4}$ and Kate Northstone ${ }^{1 *}$ \\ ${ }^{1}$ School of Social and Community Medicine, University of Bristol, Oakfield House, Oakfield Grove, Clifton, Bristol BSs \\ $2 B N, U K$ \\ ${ }^{2}$ Department of Pediatrics and Program in Graduate Medical Nutrition Sciences, Boston University School of Medicine, \\ 88 East Newton Street, Vose Hall 308, Boston, MA 02188, USA \\ ${ }^{3}$ Department of Epidemiology, Boston University School of Public Health, 88 East Newton Street, Vose Hall 308, Boston, \\ MA 02188 , USA \\ ${ }^{4}$ Program in Gastronomy, Culinary Arts, and Wine Studies, Metropolitan College at Boston University, Boston, MA 02215, USA \\ (Submitted 15 February 2012 - Final revision received 9 July 2012 - Accepted 31 July 2012 - First published online 6 September 2012)
}

\section{Abstract}

Principal components analysis (PCA) is a popular method for deriving dietary patterns. A number of decisions must be made throughout the analytic process, including how to quantify the input variables of the PCA. The present study aims to compare the effect of using different input variables on the patterns extracted using PCA on 3-d diet diary data collected from 7473 children, aged 10 years, in the Avon Longitudinal Study of Parents and Children. Four options were examined: weight consumed of each food group (g/d), energy-adjusted weight, percentage contribution to energy of each food group and binary intake (consumed/not consumed). Four separate PCA were performed, one for each intake measurement. Three or four dietary patterns were obtained from each analysis, with at least one component that described 'more healthy' and 'less healthy' diets and one component that described a diet with high consumption of meat, potatoes and vegetables. There were no obvious differences between the patterns derived using percentage energy as a measurement and adjusting weight for total energy intake, compared to those derived using gram weights. Using binary input variables yielded a component that loaded positively on reduced fat and reduced sugar foods. The present results suggest that food intakes quantified by gram weights or as binary variables both resulted in meaningful dietary patterns and each method has distinct advantages: weight takes into account the amount of each food consumed and binary intake appears to describe general food preferences, which are potentially easier to modify and useful in public health settings.

Key words: Dietary patterns: Principal components analysis: Avon Longitudinal Study of Parents and Children

The use of dietary patterns to explore the effects of diet on a variety of health outcomes is now well established as a method that complements examining individual foods and nutrients. Dietary patterns allow the assessment of the whole diet, accounting for the fact that foods/nutrients are consumed in combination and are therefore highly correlated. Principal components analysis (PCA), a form of factor analysis, is a popular method for deriving dietary patterns. It makes use of the correlations between food intakes to identify underlying patterns in the data. There are several subjective decisions that must be made when using PCA. A particularly important one, which is often overlooked, is how to quantify the input variables. Depending on the source of dietary data, a number of different variables could be considered. For example, data from diet diaries can be quantified continuously as gram weights or percentage energy from food groups or dichotomously (i.e. whether each food group was consumed or not).

The input variables used in PCA vary across studies ${ }^{(1)}$ and include frequency of consumption, gram weights, energyadjusted weight, daily percentage energy contribution and binary variables. Many studies based on diet diaries use weight of foods consumed as the input variable ${ }^{(2-5)}$. Energy adjustment using the residual method ${ }^{(6)}$ is often applied in studies based on diet diaries and diet recalls ${ }^{(7-9)}$, as well as studies based on FFQ data ${ }^{(10-12)}$. Percentage energy is another

\footnotetext{
Abbreviations: A1, A2, A3, components derived from weights (g/d) adjusted for total energy intake using the residual method; ALSPAC, Avon Longitudinal Study of Parents and Children; B1, B2, B3, B4, components derived from binary variables; P1, P2, P3, P4, components derived from percentage contribution of each food to total energy intake; PCA, principal components analysis; W1, W2, W3, components derived from weights (g/d).
} 
potential input variable (13) and a few studies ${ }^{(14,15)}$ have dichotomised intakes into binary variables. Most studies select one strategy for dietary patterns analyses, but seldom justify the decision, and only a few studies have made comparisons between the different input variables but with no formal conclusions ${ }^{(14,16,17)}$. There are no studies to our knowledge that have compared all four strategies and no studies have made comparisons in children.

In order to facilitate comparisons across studies, it is vital that researchers are as informed as possible about the decisions that they need to make and use the best evidence available. Therefore, the aim of the present study is to derive dietary patterns using PCA and using four different input variables - weight $(\mathrm{g} / \mathrm{d})$, energy-adjusted weight, percentage energy contribution and binary variables (consumed or not consumed) - and compare the interpretability of the patterns among children participating in the Avon Longitudinal Study of Parents and Children (ALSPAC).

\section{Methods \\ Participants}

The ALSPAC is an ongoing longitudinal cohort study designed to investigate determinants of development, health and disease during and after childhood. Eligible participants were pregnant women resident in the former Avon Health Authority, in South West England, due to deliver between 1 April 1991 and 31 December 1992. Further details are given elsewhere $^{(18)}$ and can be found on the website http://www.bris. ac.uk/alspac. The study includes children from the core ALSPAC sample, consisting of 14541 pregnancies, and an additional 542 eligible pregnancies not in the core sample, invited to participate at a later date. The present study was conducted according to the guidelines laid down in the Declaration of Helsinki, and all procedures involving human subjects/patients were approved by the ALSPAC Law and Ethics Committee and the Local Research Ethics Committees. Written informed consent was obtained from all subjects/ patients.

\section{Dietary assessment}

The study children were invited to attend a clinic when they were 10 years old, and a diet diary was sent with their confirmation to be completed prior to their visit. Children and their care-givers recorded, in household measures, all food and drink consumed by the child over two (not necessarily consecutive) weekdays and one weekend day. During clinic attendance, the children were interviewed to ensure the quality of the diary (e.g. clarifying portion size or omitted details on the types of food and drinks consumed). If the child did not bring a diary to the clinic, the fieldworker conducted a 24-h recall to record all food and drink consumed by the child in the previous day. Further details are given elsewhere $^{(19)}$. The completed diaries were entered into the Diet In Data Out computer program ${ }^{(20)}$, which generated the weight and energy contribution of every food consumed by each child. For the purposes of the present study, the average daily intake of food weight and energy were used.

Each food consumed was initially allocated to one of ninety-five food groups that were based on those used in FFQ that had previously been administered to the ALSPAC cohort $^{(21)}$. Sugar-free confectionery, alcohol, herbs and spices were removed from the analysis, as very few children consumed these foods and, thus, they did not contribute meaningfully to any dietary patterns. The remaining food items were combined into sixty-two groups, based on similarities between foods (e.g. nuts, peanuts and peanut butter were combined), to reduce the number of input variables and prevent infrequently consumed foods from diluting the dietary patterns. The appendix describes the food groups in detail.

\section{Statistical methods}

Dietary patterns were derived using PCA. Principal components are linear combinations of the input variables and explain as much of the variation in the data as possible. Each component describes a dietary pattern and the linear combination allows the calculation of a component score for each child; the higher the score, the more likely this pattern is present in an individual's diet. The patterns described by each component may be interpreted by its factor loadings, which are the correlations between the component and each input variable. Large positive or negative factor loadings indicate the foods that are important in that component; loadings with magnitude of at least $0 \cdot 2$ were considered when describing dietary patterns. Scree plots ${ }^{(22)}$ and the interpretability of each component were also used to determine the appropriate number of components to select. Varimax rotation ${ }^{(23)}$ was employed to aid the interpretation of components. The purpose of the present study was to compare the different dietary patterns obtained using each of the input variables; therefore, the patterns were given alphanumeric labels rather than descriptive names to aid reporting.

Four separate analyses were carried out using four different input variables. The first used the weight $(\mathrm{g} / \mathrm{d})$ of each food consumed. The variables were standardised prior to entry into the PCA to prevent components being dominated by the foods that are consumed in the highest quantities, such as water. The second analysis adjusted the mean weight for total energy intake, using the residuals method ${ }^{(6)}$. Specifically, the PCA input variables were the standardised residuals from a linear regression of mean weight on mean daily energy intake. Regression was only performed on non-zero values, and both weight and energy were log-transformed before regression and transformed back before standardisation. The third analysis used the percentage contribution of each food to the daily energy intake as input variables. These percentage energy input variables were also standardised prior to entry into the PCA to prevent the components being dominated by the foods that provide the highest percentage energy. In the fourth analysis, the input variables were dichotomised into binary variables (consumed or not consumed), as food intake variables were highly skewed and many children did 
not consume some of the food groups. The PCA was performed directly on their covariance matrix for this fourth method (as opposed to the correlation matrix for the previous three methods), as standardisation is not appropriate for binary variables. For each of the four PCA, scores were calculated for each subject, for each pattern derived by summing the products of each standardised input variable and their corresponding coefficient in the component (or dichotomised in the case of binary variables).

Agreement between the derived patterns was assessed in two ways. Agreement between component scores was assessed by calculating Pearson's sample correlation coefficients. Congruence coefficients ${ }^{(24)}$ were also calculated for pairs of matrices of component coefficients in order to assess the difference between the coefficients assigned to individual foods by each component.

\section{Results}

Of the 11868 children eligible to attend the clinic, a total of $7557(63.7 \%)$ attended and 7473 of these (98.9\%) provided dietary information. Of these, $5769(77 \cdot 2 \%)$ provided $3 \mathrm{~d}$ of dietary records. Girls, white children, children with older, more educated, non-smoking mothers and children from homes that were owned or mortgaged were more likely to provide data (all $P<0.001$; data not shown).

When gram weights were used as input variables, three principal components were retained and explained $10.4 \%$ of the variation in the sample. Factor loadings are shown in Table 1. The first component (W1) had high positive loadings on non-white bread, fruit and vegetables, cooked pasta, tuna and oily fish, cheese, yoghurt, high energy density sauce (e.g. mayonnaise), fruit juice and water. There were high negative loadings on processed meat, coated poultry, tinned pasta/ baked beans, chips (French fries), crisps (potato chips) and carbonated sweet drinks (non-diet soda). The second component (W2) had high positive loadings on meat, roast potatoes, batter/pastry products, vegetables, puddings and low energy density sauce (e.g. gravy, ketchup) and a high negative loading on chips. The third component (W3) had high positive loadings on white bread, margarine, cheese, cold meats, salty flavourings, crisps, biscuits (cookies) and diet squash/cordial.

As can be seen in Table 2, energy adjustment did not have a discernible effect on the dietary patterns when compared with those using unadjusted weights: the factor loadings were almost identical, differing by no more than $0 \cdot 084$.

Four components were obtained when percentage energy contribution was used as the input variable, explaining $12.3 \%$ of the variation in the sample. Factor loadings are shown in Table 3. The first three components, labelled P1, P2 and P3, had high loadings on the same foods that loaded highly on components W1, W2 and W3, with the exception that water loaded highly on W1 but not P1; vegetarian products, legumes and nuts loaded highly on P1 but not W1; and diet squash/cordial loaded highly on W3 but not P3. The fourth component ( $\mathrm{P} 4)$ had high positive loadings on reduced fat milk, yoghurt, breakfast cereal and biscuits and high negative loadings on rice, other breads (e.g. pitta), poultry, eggs, butter, salad, legumes and carbonated sweet drinks.

When PCA was performed on binary variables, four components were obtained, explaining $17 \cdot 3 \%$ of the variation in the sample. Table 4 shows factor loadings for these four components. The first component (B1) had high loadings on meat, roast potatoes, batter/pastry products, vegetables and low energy density sauces. The second component (B2) had high positive loadings on non-white bread, fruit, nuts, salad, vegetarian foods and vegetable dishes, potatoes, pasta, tuna and oily fish, cheese, yoghurt, eggs, butter, high energy density sauce, sweet spreads (e.g. jam), dairy puddings, cakes, chocolate, fruit juice, regular squash/cordial and water. There were high negative loadings on diet squash/cordial, and roast potatoes. The third component (B3) had high loadings on processed meat, coated poultry, tinned pasta/baked beans, white bread, margarine, vegetable oil, chips, crisps, chocolate, sweets (candy), sweet spreads (jams), sugar, cakes, dairy puddings, biscuits, carbonated sweet drinks and diet squash/cordial. The fourth component (B4) had high positive loadings on reduced fat milk, margarine, diet carbonated drinks and diet squash/cordial. It also had high negative loadings on their alternatives, i.e. full-fat milk, butter, carbonated sweet drinks and regular squash/cordial. It also had a high positive loading on breakfast cereals.

Table 5 shows the correlations between the component scores, and Table 6 shows congruence coefficients between components. The components generated from gram weights and energy-adjusted weight input variables are very similar, as assessed by correlations between component scores and the congruence coefficient between these components. The first three components from the analysis with percentage energy input variables were also similar to those generated from gram weights: the correlations among P1, P2, P3 and W1, W2, W3 were at least 0.907. The components generated by binary input variables share partial similarities with the other components. In terms of component scores, B1 was positively correlated with W2, B2 with W1 and B3 was negatively correlated with W1.

\section{Discussion}

The present study of dietary diary data from 10-year-old children compared dietary patterns derived from PCA using four strategies for quantifying input variables. When continuous variables were used (gram weights, energy-adjusted weight and percentage energy contribution), the first three components extracted had similar loadings and described similar dietary patterns: one contrasting 'more healthy' foods with 'less healthy' foods, one with high loadings on meat, potatoes and vegetables and one with high loadings on lunch and snack foods. The fourth component, present only when intake was measured as percentage energy, was difficult to interpret. When binary variables were used, the four components extracted described slightly different dietary patterns: the component with high loadings on meat, potatoes and vegetables was still present, but the component with positive loadings on 'more healthy' foods and negative loadings on 
Table 1. Factor loadings from principal components analysis of diet diary data on 7473 children aged 10 years, where input variables are weights $(\mathrm{g} / \mathrm{d})$

\begin{tabular}{|c|c|c|c|}
\hline Factor (variance explained) & W1 $(3.80 \%)$ & W2 $(3.60 \%)$ & W3 (3.10\%) \\
\hline Full-fat milk & -0.056 & 0.004 & -0.055 \\
\hline Reduced fat milk & $0 \cdot 158$ & 0.023 & -0.012 \\
\hline Cheese & $0.309^{*}$ & -0.123 & $0.261^{*}$ \\
\hline Yoghurt and fromage frais & $0.208^{*}$ & -0.030 & 0.157 \\
\hline Butter and animal fat & 0.162 & -0.081 & -0.099 \\
\hline Margarine & 0.061 & 0.035 & $0.712^{*}$ \\
\hline Vegetable oil & -0.083 & -0.058 & -0.057 \\
\hline High-fibre bread & $0.334^{*}$ & -0.099 & -0.063 \\
\hline Low-fibre bread & -0.012 & -0.040 & $0.707^{*}$ \\
\hline Other bread (e.g. pitta) & $0.223^{\star}$ & -0.077 & -0.101 \\
\hline Batter and pastry products & 0.032 & $0.277^{\star}$ & 0.081 \\
\hline Breakfast cereal & 0.098 & -0.036 & -0.175 \\
\hline Rice & 0.164 & -0.016 & -0.156 \\
\hline Pasta & $0.249^{*}$ & -0.045 & -0.086 \\
\hline Baked beans and tinned pasta & $-0.210^{*}$ & -0.117 & -0.099 \\
\hline Pizza & -0.036 & -0.176 & -0.090 \\
\hline Eggs & 0.089 & -0.074 & -0.030 \\
\hline Coated and fried chicken & $-0.310^{*}$ & -0.132 & -0.104 \\
\hline Poultry & 0.052 & $0.223^{*}$ & -0.047 \\
\hline Ham and bacon & -0.008 & 0.006 & $0.235^{\star}$ \\
\hline Red meat & 0.023 & $0.233^{*}$ & -0.056 \\
\hline Meat pies and pasties & -0.142 & 0.059 & -0.037 \\
\hline Processed meat & $-0.295^{\star}$ & -0.042 & -0.015 \\
\hline Coated and fried white fish & -0.087 & -0.087 & -0.100 \\
\hline White fish and shellfish & 0.095 & -0.026 & -0.098 \\
\hline Tuna and oily fish & $0.276^{*}$ & -0.099 & -0.042 \\
\hline Vegetarian products & $0 \cdot 190$ & -0.069 & -0.036 \\
\hline Chips (French fries) & $-0.512^{*}$ & $-0.224^{*}$ & -0.176 \\
\hline Roast potatoes & -0.149 & $0.678^{*}$ & 0.013 \\
\hline Other potatoes & 0.152 & 0.159 & -0.027 \\
\hline Root vegetables & 0.114 & $0.251^{*}$ & -0.025 \\
\hline Carrots & 0.104 & $0 \cdot 610^{*}$ & 0.002 \\
\hline Green leafy vegetables & 0.124 & $0.527^{*}$ & -0.032 \\
\hline Peas, broad beans and sweet corn & 0.031 & $0.249^{*}$ & -0.096 \\
\hline Other cooked vegetables and dishes & $0.234^{*}$ & 0.179 & -0.069 \\
\hline Salad and tomatoes & $0.443^{*}$ & -0.149 & -0.035 \\
\hline Legumes & $0.235^{\star}$ & -0.086 & -0.068 \\
\hline Soup & 0.134 & -0.081 & -0.012 \\
\hline Nuts, seeds and peanut butter & 0.193 & -0.039 & 0.041 \\
\hline Fresh fruit & $0.427^{*}$ & -0.007 & 0.048 \\
\hline Tinned and dried fruit & 0.143 & 0.007 & -0.026 \\
\hline Puddings & 0.012 & $0.240^{*}$ & -0.169 \\
\hline Dairy puddings & -0.098 & $0.218^{\star}$ & -0.180 \\
\hline Cakes & 0.095 & 0.041 & -0.053 \\
\hline Chocolate & -0.133 & -0.023 & 0.015 \\
\hline Sweets (candy) & -0.149 & -0.012 & 0.028 \\
\hline Sugar & -0.093 & 0.053 & -0.008 \\
\hline Sweet spreads (e.g. jam) & 0.105 & 0.042 & 0.174 \\
\hline Biscuits (cookies) & -0.116 & 0.040 & $0.245^{*}$ \\
\hline Crackers and crisp breads & 0.145 & -0.038 & 0.106 \\
\hline Crisps (potato chips) & $-0.207^{\star}$ & -0.035 & $0.333^{*}$ \\
\hline Low energy density sauce (e.g. gravy, ketchup) & 0.014 & $0.599^{*}$ & -0.008 \\
\hline High energy density sauce (e.g. mayonnaise) & $0.302^{*}$ & -0.138 & -0.010 \\
\hline Salty flavouring (e.g. yeast extract) & $0 \cdot 110$ & -0.035 & $0.345^{*}$ \\
\hline Water and flavoured water & $0.304^{*}$ & -0.016 & -0.128 \\
\hline Carbonated sweet drinks (soda) & $-0.246^{*}$ & -0.076 & -0.090 \\
\hline Carbonated diet drinks (diet soda) & $-0.226^{\star}$ & 0.050 & 0.079 \\
\hline Regular squash and cordial & 0.046 & -0.065 & 0.002 \\
\hline Diet squash and cordial & -0.184 & 0.083 & $0.289^{*}$ \\
\hline Fruit juice & $0.263^{*}$ & -0.055 & -0.029 \\
\hline Flavoured milk drinks & -0.034 & -0.003 & 0.006 \\
\hline Tea and coffee & -0.034 & 0.093 & 0.079 \\
\hline
\end{tabular}

W1, W2, W3, components derived from weights $(\mathrm{g} / \mathrm{d})$

${ }^{*}$ Factor loadings with magnitude greater than $0 \cdot 2$. 
Table 2. Factor loadings from principal components analysis of diet diary data on 7473 children aged 10 years, where input variables are weights $(\mathrm{g} / \mathrm{d})$ adjusted for total energy intake using the residual method

\begin{tabular}{|c|c|c|c|}
\hline Factor (variance explained) & $\mathrm{A} 1(3.80 \%)$ & A2 $(3.60 \%)$ & A3 (3.10\%) \\
\hline Full-fat milk & -0.062 & -0.014 & -0.126 \\
\hline Reduced fat milk & 0.154 & 0.022 & -0.034 \\
\hline Cheese & $0.309^{*}$ & -0.131 & $0.211^{*}$ \\
\hline Yoghurt and fromage frais & $0.202^{*}$ & -0.038 & 0.108 \\
\hline Butter and animal fat & 0.155 & -0.104 & -0.133 \\
\hline Margarine & 0.058 & 0.037 & $0.713^{*}$ \\
\hline Vegetable oil & -0.079 & -0.072 & -0.085 \\
\hline High-fibre bread & $0.333^{*}$ & -0.104 & -0.096 \\
\hline Low-fibre bread & -0.020 & -0.047 & $0.718^{*}$ \\
\hline Other bread (e.g. pitta) & $0 \cdot 224^{*}$ & -0.083 & -0.093 \\
\hline Batter and pastry products & 0.024 & $0.271^{*}$ & 0.048 \\
\hline Breakfast cereal & $0 \cdot 101$ & -0.047 & $-0.220^{*}$ \\
\hline Rice & 0.172 & -0.020 & -0.121 \\
\hline Pasta & $0.251^{*}$ & -0.041 & -0.082 \\
\hline Baked beans and tinned pasta & $-0.212^{*}$ & -0.121 & -0.111 \\
\hline Pizza & -0.039 & -0.183 & -0.100 \\
\hline Eggs & 0.091 & -0.082 & -0.051 \\
\hline Coated and fried chicken & $-0.308^{*}$ & -0.143 & -0.105 \\
\hline Poultry & 0.058 & $0.223^{*}$ & -0.025 \\
\hline Ham and bacon & -0.006 & 0.003 & $0.224^{\star}$ \\
\hline Red meat & 0.020 & $0.230^{*}$ & -0.072 \\
\hline Meat pies and pasties & -0.142 & 0.054 & -0.064 \\
\hline Processed meat & $-0.297^{*}$ & -0.048 & -0.046 \\
\hline Coated and fried white fish & -0.092 & -0.095 & -0.103 \\
\hline White fish and shellfish & 0.098 & -0.029 & -0.095 \\
\hline Tuna and oily fish & $0.269^{*}$ & -0.101 & -0.032 \\
\hline Vegetarian products & 0.187 & -0.068 & -0.026 \\
\hline Chips (French fries) & $-0.515^{\star}$ & $-0.241^{*}$ & -0.194 \\
\hline Roast potatoes & -0.148 & $0.676^{*}$ & 0.008 \\
\hline Other potatoes & 0.149 & 0.157 & -0.044 \\
\hline Root vegetables & 0.112 & $0.249^{*}$ & -0.009 \\
\hline Carrots & $0 \cdot 105$ & $0.606^{*}$ & -0.004 \\
\hline Green leafy vegetables & 0.125 & $0.521^{*}$ & -0.036 \\
\hline Peas, broad beans and sweet corn & 0.029 & $0.245^{\star}$ & -0.101 \\
\hline Other cooked vegetables and dishes & $0.240^{*}$ & 0.176 & -0.060 \\
\hline Salad and tomatoes & $0.442^{*}$ & -0.152 & -0.039 \\
\hline Legumes & $0.241^{*}$ & -0.087 & -0.054 \\
\hline Soup & 0.136 & -0.081 & -0.011 \\
\hline Nuts, seeds and peanut butter & $0 \cdot 191$ & -0.047 & 0.024 \\
\hline Fresh fruit & $0.422^{*}$ & -0.014 & 0.012 \\
\hline Tinned and dried fruit & $0 \cdot 130$ & 0.001 & -0.057 \\
\hline Puddings & 0.010 & $0.228^{*}$ & -0.180 \\
\hline Dairy puddings & -0.099 & $0 \cdot 198$ & $-0.264^{*}$ \\
\hline Cakes & 0.093 & 0.030 & -0.129 \\
\hline Chocolate & -0.142 & -0.040 & -0.052 \\
\hline Sweets (candy) & -0.155 & -0.022 & -0.016 \\
\hline Sugar & -0.096 & 0.044 & -0.083 \\
\hline Sweet spreads (e.g. jam) & 0.098 & 0.026 & $0 \cdot 132$ \\
\hline Biscuits (cookies) & -0.130 & 0.023 & 0.177 \\
\hline Crackers and crisp breads & 0.138 & -0.042 & 0.079 \\
\hline Crisps (potato chips) & $-0.217^{\star}$ & -0.048 & $0 \cdot 295^{\star}$ \\
\hline Low energy density sauce (e.g. gravy, ketchup) & 0.017 & $0.597^{*}$ & -0.016 \\
\hline High energy density sauce (e.g. mayonnaise) & $0.304^{*}$ & -0.150 & -0.012 \\
\hline Salty flavouring (e.g. yeast extract) & 0.106 & -0.027 & $0.353^{*}$ \\
\hline Water and flavoured water & $0.307^{*}$ & -0.019 & -0.109 \\
\hline Carbonated sweet drinks (soda) & $-0.255^{\star}$ & -0.095 & -0.132 \\
\hline Carbonated diet drinks (diet soda) & $-0.223^{*}$ & 0.055 & 0.080 \\
\hline Regular squash and cordial & 0.038 & -0.083 & -0.023 \\
\hline Diet squash and cordial & -0.193 & 0.091 & $0.262^{*}$ \\
\hline Fruit juice & $0.266^{*}$ & -0.079 & -0.066 \\
\hline Flavoured milk drinks & -0.035 & -0.011 & -0.042 \\
\hline Tea and coffee & -0.037 & 0.089 & 0.048 \\
\hline
\end{tabular}

$\mathrm{A1}, \mathrm{A} 2, \mathrm{~A} 3$, components derived from weights $(\mathrm{g} / \mathrm{d})$ adjusted for total energy intake using the residual method. *Factor loadings with magnitude greater than $0 \cdot 2$. 
Table 3. Factor loadings from principal components analysis of diet diary data on 7473 children aged 10 years, where input variables are percentage contribution of each food to total energy intake

\begin{tabular}{|c|c|c|c|c|}
\hline Factor (variance explained) & P1 (3.50\%) & P2 (3.20\%) & P3 (3.00\%) & P4 (2.60\%) \\
\hline Full-fat milk & -0.054 & -0.041 & -0.134 & -0.004 \\
\hline Reduced fat milk & 0.151 & 0.007 & -0.082 & $0.492^{*}$ \\
\hline Cheese & $0.306^{*}$ & -0.137 & 0.184 & 0.062 \\
\hline Yoghurt and fromage frais & $0 \cdot 203^{*}$ & -0.075 & 0.054 & $0 \cdot 258^{\star}$ \\
\hline Butter and animal fat & 0.143 & -0.099 & -0.118 & $-0.230^{\star}$ \\
\hline Margarine & 0.066 & 0.014 & $0 \cdot 720^{*}$ & 0.078 \\
\hline Vegetable oil & -0.076 & -0.086 & -0.086 & -0.038 \\
\hline High-fibre bread & $0.331^{*}$ & -0.123 & -0.106 & 0.163 \\
\hline Low-fibre bread & -0.019 & -0.025 & $0 \cdot 740^{*}$ & -0.174 \\
\hline Other bread (e.g. pitta) & $0.235^{\star}$ & -0.097 & -0.117 & $-0.340^{\star}$ \\
\hline Batter and pastry products & 0.060 & $0.207^{\star}$ & 0.013 & -0.136 \\
\hline Breakfast cereal & 0.099 & -0.043 & $-0.263^{*}$ & $0.556^{\star}$ \\
\hline Rice & 0.183 & -0.003 & -0.114 & $-0.312^{\star}$ \\
\hline Pasta & $0 \cdot 255^{*}$ & -0.013 & -0.096 & 0.060 \\
\hline Baked beans and tinned pasta & -0.172 & -0.136 & -0.071 & 0.057 \\
\hline Pizza & -0.071 & -0.174 & -0.095 & 0.074 \\
\hline Eggs & 0.084 & -0.078 & -0.049 & $-0 \cdot 214^{\star}$ \\
\hline Coated and fried chicken & $-0 \cdot 315^{\star}$ & -0.129 & -0.079 & -0.037 \\
\hline Poultry & 0.063 & $0 \cdot 246^{\star}$ & -0.035 & $-0.302^{*}$ \\
\hline Ham and bacon & -0.039 & 0.064 & 0.153 & -0.150 \\
\hline Red meat & -0.013 & $0.363^{*}$ & -0.069 & 0.032 \\
\hline Meat pies and pasties & -0.163 & 0.032 & -0.064 & 0.065 \\
\hline Processed meat & $-0.323^{*}$ & -0.029 & -0.046 & -0.071 \\
\hline Coated and fried white fish & $-0 \cdot 115$ & -0.093 & -0.073 & 0.144 \\
\hline White fish and shellfish & 0.076 & -0.048 & -0.096 & -0.047 \\
\hline Tuna and oily fish & $0.263^{*}$ & -0.109 & -0.061 & -0.050 \\
\hline Vegetarian products & $0.271^{*}$ & -0.125 & -0.008 & -0.004 \\
\hline Chips (French fries) & $-0.558^{\star}$ & $-0.210^{*}$ & -0.154 & -0.089 \\
\hline Roast potatoes & $-0 \cdot 121$ & $0.679^{*}$ & 0.009 & -0.052 \\
\hline Other potatoes & 0.146 & 0.091 & -0.045 & 0.098 \\
\hline Root vegetables & 0.192 & 0.175 & -0.011 & -0.148 \\
\hline Carrots & 0.134 & $0.588^{*}$ & -0.006 & 0.047 \\
\hline Green leafy vegetables & 0.138 & $0.534^{*}$ & -0.020 & 0.037 \\
\hline Peas, broad beans and sweet corn & 0.024 & $0.204^{\star}$ & -0.111 & 0.034 \\
\hline Other cooked vegetables and dishes & 0.160 & -0.046 & -0.073 & -0.140 \\
\hline Salad and tomatoes & $0.203^{*}$ & -0.111 & -0.065 & $-0 \cdot 208^{\star}$ \\
\hline Legumes & $0.272^{*}$ & -0.110 & -0.075 & $-0.234^{\star}$ \\
\hline Soup & 0.130 & -0.097 & -0.015 & -0.061 \\
\hline Nuts, seeds and peanut butter & $0 \cdot 204^{*}$ & -0.062 & 0.048 & 0.009 \\
\hline Fresh fruit & $0.389^{*}$ & 0.001 & -0.004 & 0.088 \\
\hline Tinned and dried fruit & $0.205^{*}$ & -0.066 & -0.037 & 0.054 \\
\hline Puddings & 0.017 & 0.189 & -0.171 & -0.050 \\
\hline Dairy puddings & -0.082 & 0.147 & $-0.249^{\star}$ & -0.033 \\
\hline Cakes & 0.084 & 0.021 & -0.145 & -0.080 \\
\hline Chocolate & -0.145 & -0.049 & -0.047 & -0.120 \\
\hline Sweets (candy) & -0.162 & -0.015 & -0.018 & -0.066 \\
\hline Sugar & -0.078 & 0.017 & -0.108 & 0.112 \\
\hline Sweet spreads (e.g. jam) & 0.086 & -0.004 & 0.130 & 0.027 \\
\hline Biscuits (cookies) & -0.120 & 0.004 & 0.159 & $0.259^{\star}$ \\
\hline Crackers and crisp breads & 0.147 & -0.077 & 0.065 & 0.054 \\
\hline Crisps (potato chips) & $-0.208^{\star}$ & -0.021 & $0 \cdot 301^{*}$ & 0.043 \\
\hline Low energy density sauce (e.g. gravy, ketchup) & 0.082 & $0.407^{\star}$ & -0.047 & -0.043 \\
\hline High energy density sauce (e.g. mayonnaise) & $0 \cdot 279^{*}$ & -0.163 & -0.020 & -0.141 \\
\hline Salty flavouring (e.g. yeast extract) & $0 \cdot 105$ & -0.009 & $0.394^{*}$ & -0.013 \\
\hline Water and flavoured water & 0.005 & 0.029 & -0.043 & -0.050 \\
\hline Carbonated sweet drinks (soda) & $-0.280^{\star}$ & -0.098 & -0.087 & $-0 \cdot 255^{\star}$ \\
\hline Carbonated diet drinks (diet soda) & $-0.220^{*}$ & $0 \cdot 138$ & 0.074 & -0.062 \\
\hline Regular squash and cordial & 0.012 & -0.065 & 0.024 & -0.036 \\
\hline Diet squash and cordial & -0.071 & 0.033 & 0.068 & 0.165 \\
\hline Fruit juice & $0.271^{*}$ & -0.088 & -0.052 & -0.091 \\
\hline Flavoured milk drinks & -0.022 & -0.022 & -0.057 & -0.011 \\
\hline Tea and coffee & 0.012 & -0.010 & -0.043 & -0.052 \\
\hline
\end{tabular}

P1, P2, P3, P4, components derived from percentage contribution of each food to total energy intake. * Factor loadings with magnitude greater than $0 \cdot 2$. 
Table 4. Factor loadings from principal components analysis of diet diary data on 7473 children aged 10 years, where intakes are expressed as binary (consumed/not consumed) variables

\begin{tabular}{|c|c|c|c|c|}
\hline Factor (variance explained) & B1 $(5 \cdot 20 \%)$ & B2 $(5.00 \%)$ & B3 $(3.90 \%)$ & B4 (3.20\%) \\
\hline Full-fat milk & 0.065 & 0.043 & $0 \cdot 106$ & $-0.654^{*}$ \\
\hline Reduced fat milk & -0.032 & 0.077 & -0.002 & $0.773^{*}$ \\
\hline Cheese & -0.091 & $0.424^{*}$ & 0.025 & 0.089 \\
\hline Yoghurt and fromage frais & -0.019 & $0.264^{*}$ & 0.057 & 0.200 \\
\hline Butter and animal fat & -0.030 & $0.298^{*}$ & -0.069 & $-0.282^{*}$ \\
\hline Margarine & 0.086 & -0.080 & $0.230^{*}$ & $0.320^{*}$ \\
\hline Vegetable oil & -0.088 & 0.171 & $0.313^{*}$ & -0.009 \\
\hline High-fibre bread & -0.068 & $0.341^{*}$ & -0.109 & 0.112 \\
\hline Low-fibre bread & 0.045 & 0.042 & $0.230^{*}$ & 0.040 \\
\hline Other bread (e.g. pitta) & -0.001 & $0 \cdot 171$ & -0.060 & -0.020 \\
\hline Batter and pastry products & $0.392^{*}$ & -0.003 & 0.132 & -0.029 \\
\hline Breakfast cereal & 0.015 & 0.164 & 0.089 & $0.204^{\star}$ \\
\hline Rice & 0.050 & 0.173 & -0.120 & -0.014 \\
\hline Pasta & -0.052 & $0.348^{*}$ & -0.147 & 0.075 \\
\hline Baked beans and tinned pasta & -0.053 & -0.064 & $0.386^{*}$ & -0.047 \\
\hline Pizza & -0.151 & 0.101 & 0.154 & 0.029 \\
\hline Eggs & -0.035 & $0.249^{*}$ & 0.078 & -0.017 \\
\hline Coated and fried chicken & -0.070 & -0.099 & $0.386^{*}$ & -0.043 \\
\hline Poultry & $0.369^{*}$ & 0.032 & 0.029 & 0.024 \\
\hline Ham and bacon & 0.084 & 0.097 & 0.128 & 0.094 \\
\hline Red meat & $0.425^{\star}$ & 0.032 & -0.056 & 0.023 \\
\hline Meat pies and pasties & 0.083 & -0.066 & 0.080 & 0.006 \\
\hline Processed meat & 0.039 & -0.092 & $0.368^{*}$ & -0.030 \\
\hline Coated and fried white fish & -0.027 & -0.013 & 0.147 & -0.018 \\
\hline White fish and shellfish & -0.012 & 0.146 & -0.044 & -0.014 \\
\hline Tuna and oily fish & -0.047 & $0.306^{*}$ & -0.079 & 0.040 \\
\hline Vegetarian products & -0.080 & $0.203^{*}$ & -0.069 & -0.019 \\
\hline Chips (French fries) & -0.096 & -0.187 & $0.551^{*}$ & -0.091 \\
\hline Roast potatoes & $0.761^{*}$ & $-0.210^{*}$ & 0.040 & -0.053 \\
\hline Other potatoes & $0 \cdot 166$ & $0.238^{*}$ & 0.028 & 0.022 \\
\hline Root vegetables & $0.233^{*}$ & 0.181 & -0.078 & -0.011 \\
\hline Carrots & $0 \cdot 700^{*}$ & 0.062 & -0.074 & -0.022 \\
\hline Green leafy vegetables & $0.579^{*}$ & 0.078 & -0.113 & -0.025 \\
\hline Peas, broad beans and sweet corn & $0.368^{*}$ & 0.098 & 0.057 & 0.013 \\
\hline Other cooked vegetables and dishes & $0.343^{*}$ & $0.266^{*}$ & -0.135 & 0.034 \\
\hline Salad and tomatoes & -0.069 & $0.594^{*}$ & -0.086 & -0.001 \\
\hline Legumes & -0.031 & 0.190 & -0.090 & -0.034 \\
\hline Soup & -0.017 & 0.137 & -0.048 & -0.025 \\
\hline Nuts, seeds and peanut butter & -0.043 & $0.236^{*}$ & -0.023 & 0.012 \\
\hline Fresh fruit & 0.041 & $0.459^{*}$ & 0.010 & 0.082 \\
\hline Tinned and dried fruit & 0.011 & $0.298^{*}$ & 0.021 & 0.003 \\
\hline Puddings & 0.193 & 0.123 & 0.064 & -0.040 \\
\hline Dairy puddings & $0 \cdot 157$ & $0.227^{\star}$ & $0.292^{*}$ & -0.045 \\
\hline Cakes & 0.054 & $0.267^{*}$ & $0.243^{*}$ & -0.020 \\
\hline Chocolate & -0.021 & $0.210^{*}$ & $0.349^{*}$ & -0.031 \\
\hline Sweets (candy) & 0.011 & 0.137 & $0.367^{\star}$ & -0.054 \\
\hline Sugar & -0.013 & 0.129 & $0.342^{*}$ & 0.051 \\
\hline Sweet spreads (e.g. jam) & -0.044 & $0.299^{*}$ & $0.254^{\star}$ & -0.022 \\
\hline Biscuits (cookies) & 0.062 & 0.127 & $0.222^{*}$ & 0.067 \\
\hline Crackers and crisp breads & -0.016 & $0 \cdot 170$ & 0.043 & 0.021 \\
\hline Crisps (potato chips) & 0.048 & -0.015 & $0.216^{*}$ & 0.063 \\
\hline Low energy density sauce (e.g. gravy, ketchup) & $0.507^{*}$ & 0.040 & 0.085 & 0.011 \\
\hline High energy density sauce (e.g. mayonnaise) & -0.082 & $0.362^{*}$ & -0.058 & 0.037 \\
\hline Salty flavouring (e.g. yeast extract) & 0.010 & 0.123 & 0.000 & 0.017 \\
\hline Water and flavoured water & 0.015 & $0.336^{*}$ & -0.140 & -0.053 \\
\hline Carbonated sweet drinks (soda) & -0.020 & 0.050 & $0.213^{*}$ & $-0.321^{\star}$ \\
\hline Carbonated diet drinks (diet soda) & 0.036 & -0.127 & $0.252^{*}$ & $0.241^{\star}$ \\
\hline Regular squash and cordial & -0.070 & $0.241^{*}$ & 0.123 & $-0.258^{\star}$ \\
\hline Diet squash and cordial & 0.123 & $-0.244^{*}$ & $0.296^{*}$ & $0.346^{*}$ \\
\hline Fruit juice & -0.043 & $0.410^{*}$ & 0.049 & 0.007 \\
\hline Flavoured milk drinks & 0.030 & 0.122 & $0 \cdot 129$ & 0.017 \\
\hline Tea and coffee & 0.074 & -0.031 & 0.121 & 0.067 \\
\hline
\end{tabular}

B1, B2, B3, B4, components derived from binary variables.

${ }^{*}$ Factor loadings with magnitude greater than 0.2 . 
Table 5. Correlations between component scores obtained from different input variables*

\begin{tabular}{|c|c|c|c|c|c|c|c|c|}
\hline & W1 & W2 & W3 & & B1 & B2 & B3 & B4 \\
\hline A1 & 0.995 & 0.105 & -0.066 & & 0.143 & 0.652 & -0.430 & 0.159 \\
\hline A2 & 0.101 & 0.996 & -0.040 & & 0.765 & 0.058 & -0.052 & 0.075 \\
\hline A3 & -0.051 & -0.059 & 0.962 & & -0.043 & -0.151 & 0.050 & 0.241 \\
\hline P1 & 0.931 & 0.142 & -0.023 & & 0.159 & 0.599 & -0.413 & 0.199 \\
\hline P2 & 0.061 & 0.918 & -0.050 & & 0.708 & 0.010 & -0.086 & 0.068 \\
\hline P3 & -0.056 & -0.084 & 0.907 & & -0.069 & -0.154 & 0.029 & 0.160 \\
\hline P4 & 0.003 & -0.010 & -0.076 & & 0.044 & 0.043 & -0.006 & -0.392 \\
\hline B1 & 0.145 & 0.767 & -0.026 & & $\mathrm{P} 1$ & P2 & P3 & P4 \\
\hline B2 & 0.653 & 0.074 & -0.102 & $\mathrm{~A} 1$ & 0.942 & 0.065 & -0.063 & 0.004 \\
\hline B3 & -0.420 & -0.037 & 0.119 & $\mathrm{~A} 2$ & 0.144 & 0.937 & -0.078 & -0.018 \\
\hline B4 & 0.156 & 0.061 & 0.219 & A3 & -0.011 & -0.034 & 0.962 & -0.045 \\
\hline
\end{tabular}

${ }^{*} \mathrm{~W}$, components derived from weights $(\mathrm{g} / \mathrm{d})$; $\mathrm{A}$, components derived from weights $(\mathrm{g} / \mathrm{d})$ adjusted for total energy intake using the residual method; $P$, components derived from percentage contribution of each food to total energy intake; $\mathrm{B}$, components derived from binary variables.

'less healthy' foods was replaced by two components: one with high loadings on the 'more healthy' foods and the other with high loadings on the 'less healthy' foods. The fourth component had positive loadings for reduced-fat and reduced-sugar foods and negative loadings on their alternatives.

There are strong similarities between patterns in the presence and absence of energy adjustment, the main differences being in the relative loadings of high- and low-fibre bread, and full- and low-fat milk. In a comparison of energy-adjusted and unadjusted analyses of data from FFQ administered to the ALSPAC mothers ${ }^{(16)}$, five components appear in the unadjusted analysis, but four components suffice under energy adjustment; the missing component described a 'processed' dietary pattern. A study ${ }^{(17)}$ comparing gram weights and percentage energy as input variables, in PCA of FFQ data from Irish adults, concludes that gram weights give more interpretable patterns than percentage energy.

In the present study, the patterns obtained when gram weights were used as the input variables were the most interpretable. Weight is a clear, quantitative way to measure food consumption and can be easily linked to portion sizes. A drawback of using gram weights (unadjusted and adjusted for energy) and percentage energy was that they potentially led to skewed input variables, with many zeroes for foods that were not frequently consumed. This resulted in component scores with skewed distributions. Adjusting the weight for energy intake did not alter the dietary patterns, agreeing with research in adults ${ }^{(14)}$. These results suggest that energy-adjusting the input variables does not offer any specific benefit when determining dietary patterns, using PCA, from diet diaries administered to children. It may be more appropriate to perform energy adjustment later in the analytic process, as this allows for more accurate assessment of the effect of energy itself. A similar conclusion was reached when obtaining dietary patterns using PCA in the ALSPAC mothers, although this was based on the FFQ data ${ }^{(16)}$.

In agreement with other research (in adults) ${ }^{(17)}$, using percentage energy as an input variable led to patterns that were harder to interpret than those derived from gram weights.
In the present study, the percentage energy strategy led to components in which water did not load highly, as it does not contribute to energy intake. This could be considered an inherent limitation of this approach, given non-energycontaining foods (e.g. water, coffee, tea and diet soda) often contribute meaningfully to dietary patterns. This is shown in the present study, in which water loaded highly on the components obtained when gram weights were used as the input variable strategy, whether energy-adjusted or unadjusted. These results indicate that variation in water intake is an important part of childhood diet and is missed when using the percentage energy method. Percentage energy is an attractive concept, as it considers one's overall dietary composition. However, it is harder to comprehend when dealing with individual food groups, which provide relatively small contributions to total energy intake when considered on their own (i.e. in contrast to considering, say, the macronutrient composition of the diet).

Few studies have used binary input variables to derived dietary patterns using PCA. Using this method, they overcame the issues of skewness and the sometimes large numbers of non-consumers of food groups, and led to interpretable dietary patterns. A study of data from an FFQ administered to adults in four European cohorts ${ }^{(14)}$ showed no effect of dichotomisation of input variables on dietary patterns. However, in the present study, the patterns were different from those obtained from continuous variables; binary

Table 6. Congruence coefficients between components obtained from different input variables*

\begin{tabular}{lll}
\hline First set & Second set & Congruence \\
\hline & & \\
W1, W2, W3 & A1, A2, A3 & 0.994 \\
W1, W2, W3 & P1, P2, P3 & 0.954 \\
W1, W2, W3 & B2, B1, B3 & 0.624 \\
A1, A2, A3 & P1, P2, P3 & 0.964 \\
A1, A2, A3 & B2, B1, B3 & 0.579 \\
P1, P2, P3, P4 & B2, B1, B3, B4 & 0.505
\end{tabular}

${ }^{*} \mathrm{~W}$, components derived from weights $(\mathrm{g} / \mathrm{d})$; $\mathrm{B}$, components derived from binary variables; $A$, components derived from weights $(\mathrm{g} / \mathrm{d})$ adjusted for total energy intake using the residual method; $\mathrm{P}$, components derived from percentage contribution of each food to total energy intake. 
(consumed/not consumed) variables are easy to understand and conceptually represent choices and/or preferences of food rather than quantities consumed. This was evident in component B4, which seemed to differentiate among individuals who chose reduced fat, reduced sugar foods and those who chose the regular (full fat, full sugar) options for those foods. Food choices are potentially easier to modify, but it must be recognised that people consume food in different quantities, and dichotomising food intakes does not capture the complexity of eating behaviour.

The findings of the present study are strengthened by the large sample size. However, the sample is biased towards higher socio-economic status. As well, the present study has not assessed the effect of different input variables on a specific diet-disease association. As the patterns obtained with different strategies were similar, the effect of input variables on a given diet-disease association may be similar, although this is an important next step to further this literature and needs to be examined. Another input variable that could be considered is the number of servings per day, which is commonly used in studies that assess diet using an FFQ. However, as the present study made use of diet diaries, considered a 'gold standard' method of self-reported dietary assessment, we elected not to consider this semi-quantitative approach commonly used in FFQ, given the level of detail we have in the diet diaries.

In conclusion, the present study is the first to comprehensively compare different input variables used in dietary pattern analysis obtained using PCA. The present results indicate that there appears to be no benefit associated with energy adjustment, given results were similar to those when unadjusted. We also showed that patterns based on percentage energy did not capture meaningful dietary intakes, completely missing some items consumed such as water, and were also harder to interpret. Thus, while the final choice of input variable treatment may depend on the purpose of a particular analysis, the use of food weights and binary variables appeared to be the best approaches to quantify input variables in the present study among children. More research is needed to see whether input variable treatment has an impact on diet-disease associations, as understanding the role of diet on health outcomes is the ultimate objective of nutritional epidemiological studies. However, for the purposes of describing the underlying patterns of diet in a population, we would recommend using weights of foods; binary input variables would be a complementary approach to this in which specific dietary choices can be identified.

\section{Acknowledgements}

We are extremely grateful to all the families who took part in the present study, the midwives for their help in recruiting them and the whole ALSPAC team, which includes interviewers, computer and laboratory technicians, clerical workers, research scientists, volunteers, managers, receptionists and nurses. The UK Medical Research Council, the Wellcome Trust and the University of Bristol provide core support for ALSPAC. The present work was supported by the World Cancer Research Fund grant number 2009/23.
K. N. and P. M. E. designed the study; A. D. A. C. S. performed the statistical analysis; K. N. had primary responsibility for final content. All authors contributed to writing the manuscript and approved the final version. The authors declare no conflict of interest.

\section{References}

1. Newby PK \& Tucker KL (2004) Empirically derived eating patterns using factor or cluster analysis: a review. Nutr Rev 62, 177-203.

2. Cucó G, Fernández-Ballart J, Sala J, et al. (2006) Dietary patterns and associated lifestyles in preconception, pregnancy and postpartum. Eur J Clin Nutr 60, 364-371.

3. Hamer M, McNaughton SA, Bates CJ, et al. (2010) Dietary patterns, assessed from a weighed food record, and survival among elderly participants from the United Kingdom. Eur J Clin Nutr 64, 853-861.

4. Mikkilä V, Räsänen L, Raitakari OT, et al. (2005) Consistent dietary patterns identified from childhood to adulthood: The Cardiovascular Risk in Young Finns Study. Br J Nutr 93, 923-931.

5. Yannakoulia M, Yiannakouris N, Melistas L, et al. (2008) A dietary pattern characterized by high consumption of whole-grain cereals and low-fat dairy products and low consumption of refined cereals is positively associated with plasma adiponectin levels in healthy women. Metabolism $\mathbf{5 7 ,} 824-830$.

6. Willett WC, Howe GR \& Kushi LH (1997) Adjustment for total energy intake in epidemiologic studies. Am J Clin Nutr $\mathbf{6 5}$, Suppl., 1220S-12288S.

7. Kesse-Guyot E, Vergnaud A, Fezeu L, et al. (2010) Associations between dietary patterns and arterial stiffness, carotid artery intima-media thickness and atherosclerosis. Eur $J$ Cardiovasc Prev Rehabil 17, 718-724.

8. McNaughton SA, Mishra GD, Bramwell G, et al. (2005) Comparability of dietary patterns assessed by multiple dietary assessment methods: results from the 1946 British Birth Cohort. Eur J Clin Nutr 59, 341-352.

9. Okubo H, Murakami K, Sasaki S, et al. (2010) Relative validity of dietary patterns derived from a self-administered diet history questionnaire using factor analysis among Japanese adults. Public Health Nutr 13, 1080-1089.

10. Bamia C, Orfanos P, Ferrari P, et al. (2005) Dietary patterns among older Europeans: the EPIC - Elderly study. $\mathrm{Br} J$ Nutr 94, 100-113.

11. Martínez-Ortiz JA, Fung TT, Baylin A, et al. (2005) Dietary patterns and risk of nonfatal acute myocardial infarction in Costa Rican adults. Eur J Clin Nutr 60, 770-777.

12. Velie EM, Schairer C, Flood A, et al. (2005) Empirically derived dietary patterns and risk of postmenopausal breast cancer in a large prospective cohort study. Am J Clin Nutr 82, 1308-1319.

13. Newby PK, Muller D, Hallfrisch J, et al. (2004) Food patterns measured by factor analysis and anthropometric changes in adults. Am J Clin Nutr 80, 504-513.

14. Balder HF, Virtanen M, Brants HAM, et al. (2003) Common and country-specific dietary patterns in four European cohort studies. J Nutr 133, 4246-4251.

15. Guinot C, Latreille J, Malvy D, et al. (2001) Use of multiple correspondence analysis and cluster analysis to study dietary behaviour: food consumption questionnaire in the SU.VI.MAX. cohort. Eur J Epidemiol 17, 505-516.

16. Northstone K, Ness AR, Emmett PM, et al. (2008) Adjusting for energy intake in dietary pattern investigations using principal components analysis. Eur J Clin Nutr 62, 931-938. 
17. Hearty AP \& Gibney MJ (2009) Comparison of cluster and principal components analysis techniques to derive dietary patterns in Irish adults. Br J Nutr 101, 598-608.

18. Golding J, Pembrey M, Jones R, et al. (2001) ALSPAC - The Avon Longitudinal Study of Parent and Children. I. Study methodology. Paediatr Perinat Epidemiol 15, 74-87.

19. Cribb VL, Jones LR, Rogers IS, et al. (2011) Is maternal education level associated with diet in 10-year-old children? Public Health Nutr 14, 2037-2048.

20. Price GM, Paul AA, Key FB, et al. (1995) Measurement of diet in a large national survey: comparison of computerised and manual coding of records in household measures. I Hum Nutr Diet 8, 417-428.

21. Northstone K, Emmett P \& The ALSPAC Study Team (2005) Multivariate analysis of diet in children at four and seven years of age and associations with socio-demographic characteristics. Eur J Clin Nutr 59, 751-760.

22. Cattell RB (1966) The scree test for the number of factors. Multivariate Behav Res 1, 245-276.

23. Kline P (1994) An Easy Guide to Factor Analysis. London: Routledge.

24. Harman HH (1976) Modern Factor Analysis. Chicago, IL: University of Chicago Press. 
Appendix: Food groups and their components

Full-fat milk

Reduced fat milk

Cheese

Yoghurt and fromage frais

Butter and animal fat

Margarine

Vegetable oil

High-fibre bread

Low-fibre bread

Other bread

Batter and pastry products

Breakfast cereal

Rice

Pasta

Baked beans and tinned pasta

Pizza

Eggs

Coated and fried chicken

Poultry

Ham and bacon

Red meat

Meat pies and pasties

Processed meat

Coated and fried white fish

White fish and shellfish

Tuna and oily fish

Vegetarian products

Chips (French fries)

Roast potatoes

Other potatoes

Root vegetables

Carrots

Green leafy vegetables

Peas, broad beans and sweet corn

Other cooked vegetables and dishes

Salad and tomatoes

Legumes

Soup

Nuts, seeds and peanut butter

Fresh fruit

Tinned and dried fruit

Puddings

Dairy puddings

Cakes

Chocolate

Sweets (candy)

Sugar

Sweet spreads

Biscuits (cookies)

Crackers and crisp breads

Crisps (potato chips)

Low energy density sauce

High energy density sauce

Salty flavouring

Water and flavoured water

Carbonated sweet drinks (soda)

Carbonated diet drinks (diet soda)

Regular squash and cordial

Diet squash and cordial

Fruit juice

Flavoured milk drinks

Tea and coffee

Foods not included $\ddagger$
Full-fat cow's, sheep's or goat's milk

Skimmed or semi-skimmed cow's milk

Hard, soft, cream or cottage cheese

Plain or fruit yoghurt, fromage frais

Butter, dripping, ghee, lard, suet

Hard or soft margarine or spread

Canola/rapeseed, coconut, cod liver, corn, olive, peanut, safflower, sesame, soya or sunflower oil

White bread, hamburger buns, bagels

Brown, wholemeal, granary or rye bread

Pitta or naan bread, ciabatta, chapattis, papadums, tortillas

Breadcrumbs, brioche, croissants, pancakes, pastry, scones, stuffing, Yorkshire pudding

Bran, corn, rice or oat-based cereal or sweetened cereal

Brown, white, risotto or pilau rice

Pasta, spaghetti, macaroni, lasagna, noodles, couscous

Baked beans, canned spaghetti or ravioli, macaroni cheese, pasta salad, gnocchi, cannelloni, pot snacks

Pizza, lunchbox snacks

Hen's, duck's or quail's eggs, quiche, omelette, Scotch eggs

Chicken or turkey burgers, fingers, Kiev, nuggets or in crumbs

Chicken, turkey, duck, rabbit, grouse, pheasant

Ham, gammon, bacon

Beef, lamb, pork, veal, venison, haggis, liver, kidney

Beef, chicken or pork pie, sausage rolls

Sausages, burgers, luncheon meat

Cod, haddock, plaice, skate all in batter or breadcrumbs

Cod, coley, haddock, hake, halibut, monkfish, plaice, sea bass, snapper, sole, clams, crab, cockles, mussels, scallops, scampi, squid, prawns

Tuna, anchovies, herring, kipper, mackerel, pilchards, salmon, sardines, swordfish, trout

Vegetable or bean burgers/sausages, Quorn, soya

Chips, fried potatoes, potato waffles or croquettes

Old potatoes, roasted in fat

New and old potatoes, boiled or baked

Artichoke, beetroot, garlic, onion, parsnip, swede, turnip, yam

Carrots

Broccoli, Brussels sprouts, cabbage, kale, spinach

Peas, broad beans, sweet corn, mange-tout

Asparagus, cauliflower, celery, courgette, green or French beans, leek, marrow,

peppers, pumpkin, squash, vegetable flans or pastries, cauliflower cheese

Raw vegetables, tomatoes

Beans, lentils

Soup

Nuts, peanuts, seeds, peanut butter

Citrus or other fruit

Tinned or dried fruit

Cheesecake, Christmas pudding, crumble, flan, fruit pie, jelly, Pavlova, sponge, trifle

Blancmange, bread and butter pudding, cream, custard, ice cream, mousse, rice pudding

Buns, cakes, pastries

Chocolate confectionery

Sugar confectionery

Sugar, icing

Jam, honey, chocolate spread, lemon curd, marmalade

Biscuits, fully-coated chocolate biscuits

Crackers, oatcakes, water biscuits, cheese biscuits, rice cakes

Potato crisps, corn snacks, pretzels

Bread/cheese/tomato sauces, gravy, mustard, vinegar. Energy density below $8 \mathrm{~kJ} / \mathrm{g}(2 \mathrm{kcal} / \mathrm{g})$

Mayonnaise, salad cream, chutney. Energy density above $8 \mathrm{~kJ} / \mathrm{g}(2 \mathrm{kcal} / \mathrm{g})$

Yeast extract, stock cubes, table salt

Water, flavoured water

Cola, lemonade, ginger ale, tonic water, energy drinks

Diet cola, lemonade or energy drinks

Fruit squash or cordial ${ }^{*}$

Low sugar fruit squash or cordial ${ }^{\star}$

Fruit juice

Flavoured milk

Tea, coffeet, herbal tea

Sugar-free sweets/jelly/mints/chewing gum, artificial sweetener, black treacle,

instant dessert powder, diabetic jam/chocolate

Alcoholic drinks

Herbs, spices

* Weight of undiluted squash was multiplied by five to obtain equivalent diluted weight.

† Weight of coffee granules was multiplied by 190 to obtain equivalent liquid weight.

$\ddagger$ Due to infrequency of consumption and lack of importance in any extracted component. 